Journal of Case Reports 2017;7(2):211-213

\title{
Neurothekeoma Thumb
}

\author{
Reshmi Narayanan Raju, V Kunjambu Nambiar, Arun Kumar, John Thomas \\ Department of General Surgery, Pariyaram Medical College, Kannur, Kerala - 670503, India.
}

\section{Corresponding Author:}

Dr. Reshmi Narayanan Raju

Email: rajureshmi5@gmail.com

This is an Open Access article distributed under the terms of the Creative Commons Attribution License (creativecommons.org/ licenses/by/3.0).

Received : February 25, 2017

Accepted : May 3, 2017

Published : June 10, 2017

\begin{abstract}
Background: Neurothekeomas are rare benign neoplasms typically occurring in young female patients. The usual presentations are small nodules in different anatomical sites commonly involving the face and the upper limb. Case Report: Here we describe a 52 year-old male who had a swelling in the left thumb for 2 years. Histology showed lobulated mass within the dermis containing spindle cells and abundant myxoid matrix. Immunohistochemistry was positive for S100. These findings were compatible with myxoid neurothekeoma. The lesion was completely excised and at the 6-month followup the patient was asymptomatic and had no recurrences. Conclusion: This case of neurothekeoma is an exception as the presentation involves thumb involvement which opined as myxoid neurothekeoma and no recurrence of tumor was reported on follow-up.
\end{abstract}

Keywords: Dermis, Upper Extremity, Thumb, Immunohistochemistry, Neurothekeoma.

\section{Introduction}

Neurothekeoma is a benign, predominantly cutaneous neoplasm that was initially described by Harkin and Reed in 1969 by the name 'nerve sheath myxoma' [1]. In 1980 Gallager and Helwig termed the lesion 'Neurothekeoma' (Greek: theke, sheath) to imply both the histologic nested appearance and the nerve sheath differentiation [2]. There are overlapping feature with other neural tissue tumors such as schwannoma, nerve sheath myxoma and neurofibroma leading to difficulty in diagnosis.

Neurothekeoma's most commonly occur in the head and neck. The nose and scalp are the most frequent sites of involvement, followed by the orbital region, cheeks, and chin [3]. The upper and lower extremities and trunk are other commonly reported sites of involvement. On clinical examination, neurothekeoma's appear asymptomatic to mildly tender, flesh-colored solitary nodules less than $2 \mathrm{~cm}$. Sometimes an overlying erythema may be present. They may be mistaken for epidermal inclusion cysts, intradermal nevi, lipomas, pilomatrixomas, or dermatofibromas.
Histologically neurothekeomas consist of spindled and epithelioid cells with abundant eosinophilic, finely granular cytoplasm. The cells are situated in multiple, closely spaced nodules of varying sizes within the dermis. Although considered a benign neoplasm, due to the possibility of recurrence and local invasion, it is recommended that wide local excision with frozensection-margin control be employed for treatment [4].

\section{Case Report}

A 52 year-old healthy male with no known comorbidities presented with a 2-year history of an asymptomatic lesion in the palmar aspect of left thumb. Initially the swelling was in the size of a peanut and then gradually increased in size to reach the present size. It was not associated with pain. No history of trauma was present. On examination, a firm non-tender swelling of size $4 \times 2 \mathrm{~cm}$ over palmar aspect of left thumb distal to distal inter-phalangeal joint was present. Swelling was freely mobile over underlying structures, no pulsation was present and skin over swelling 
was not pinchable. Systemic examination was unremarkable. Complete excision of swelling along with skin and Z-plasty was done.

Histopathology report opined as lobulated mass within the dermis. The tumor lobules were variably sized separated by thin fibro-vascular septae and composed of stellate and spindle cells in an abundant myxoid matrix. No atypical cells or mitotic figures were seen [Fig.1,2]. Immunohistochemistry was positive for S-100. At 6 months follow up, the patient was asymptomatic without recurrence signs and excellent healing process.

\section{Discussion}

Neurothekeoma through the years have been variously described as 'Bizarre cutaneous neurofibroma', 'Myxoma of nerve sheath' and 'Pacinian neurofibroma' [5]. The average patient age is about 25 years, but neurothkeomas may occur at any age [3]. Women are affected more often, the male to female ratio is approximately 1:2 [3]. Local trauma and estrogen use have been identified as triggering factors $[6,7]$.

They are often solitary and may be either asymptomatic or may present as a painful raised skin-colored well-circumscribed dome-shaped nodule less than $3 \mathrm{~cm}$ in diameter. Based on histopathological appearance and immunohistochemical findings, there are three variants of neurothekeoma-myxoid (classical or hypocellular), cellular and mixed type [8]. The myxoid neurothekeoma is characterized by greater degree of myxomatous changes, less cellularity with wellcircumscribed spindle cells in myxoid matrix and multinucleated giant cells are consistently positive for S-100 proteins. Hence S-100 is considered to be a sensitive immunohistochemical marker for neurothekeoma. They are also known to be positive for vimentin but negative for cytokeratin, desmin, CD34, SMA (smooth muscle actin). In contrast, the cellular types of neurothekeoma are not encapsulated. The cells are epithelioid with

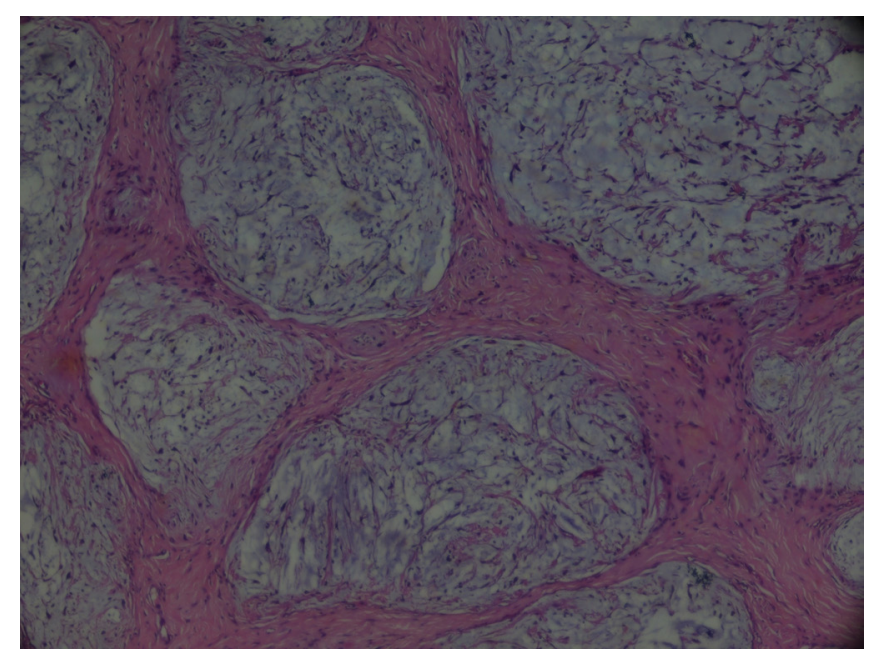

Fig.1: Lobulated mass within the dermis separated by myxoid matrix.

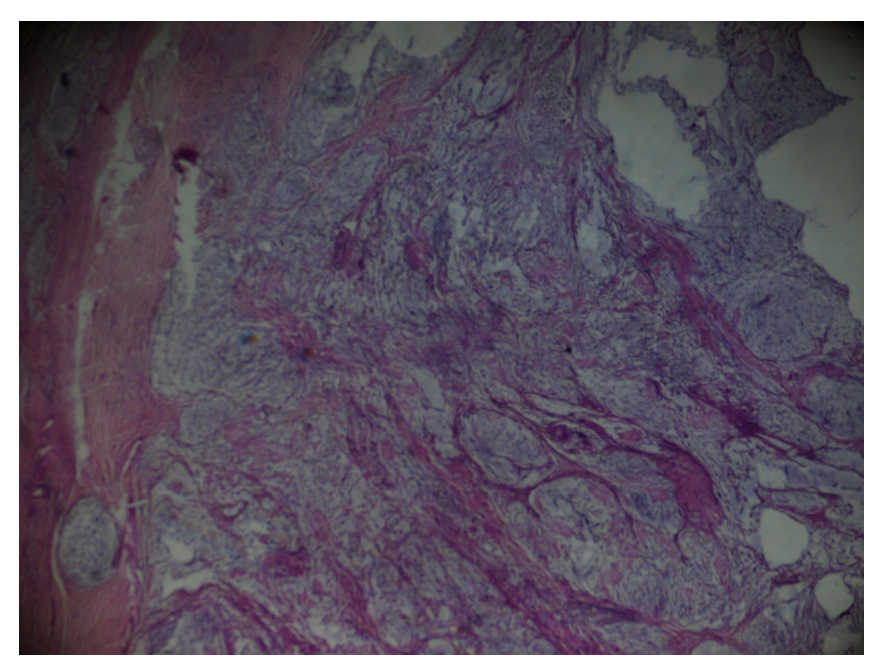

Fig.2: Lobules composed of stellate and spindle cells surrounded by matrix.

eosinophilic cytoplasm, they do not stain S-100, collagen type IV or nerve growth factor receptor but show reactivity with NK1C3 (CD57) and the panmonocyte marker Ki-M1p [9]. The mixed type of neurothekeoma shows areas of varied cellularity with focal myxoid regions.

The cell of origin of neurothekeoma is controversial with theories proposing a proliferation of Schwann cells or other perineural cells [10]. Dual immunoreactivity for NSE and S-100 protein in a myxoid background supports 
Schwann cell origin, whereas the absence of S-100 protein and positive epithelial membrane antigen in the cellular type suggests perineural cell lineage [10]. Fetsch et al. [3] postulated a tendency of neurothekeoma to recruit histiocytic cells and to differentiate into myofibroblasts. Smooth muscle actin (SMA) positivity suggests some similarity to the epithelioid variant of leiomyoma or myofibroblastic proliferations [11]. Glial fibrillary acidic protein (GFAP) positivity is rare in neurothekeoma; however, its positivity further supports a peripheral nerve sheath origin, as occasional peripheral nerve sheath tumors may coexpress GFAP and S-100 [12]. Complete excision is the mainstay of treatment for neurothekeoma. Recurrence of the tumor may be due to incomplete removal of the original lesion [13]. Our patient was followed up for six months with no reports of recurrence.

\section{Conclusion}

This case of neurothekeoma is an exception as the presentation involving thumb which opined as myxoid neurothekeoma and on follow up period no recurrence of tumor was reported.

Contributors: RNR: Manuscript writing, case management; VKN: manuscript revision, case management; AK, JT: critical review of manuscript for intellectual content, case management. RNR will act as guarantor. All authors approved final version of the manuscript.

Funding: None; Competing interests: None stated.

\section{References}

1. Tumors of the peripheral nervous system - NLM Catalog - NCBI. Available from: https://www.ncbi.nlm.nih.gov/ nlmcatalog/101140625. Accessed on Feburary 25, 2017.
2. Gallager RL, Helwig EB. Neurothekeoma a benign cutaneous tumor of neural origin. Am J Clin Pathol. 1980;74:759-764.

3. Fetsch JF, Laskin WB, Hallman JR, Lupton GP, Miettinen M. Neurothekeoma: An analysis of 178 tumors with detailed immunohistochemical data and long-term patient follow-up information. Am J Surg Pathol. 2007;31:1103-1114.

4. Ward JL, Prieto VG, Joseph A, Chevray P, Kronowitz S, Sturgis EM. Neurothekeoma. Otolaryngol - Head Neck Surg. 2005; 132:86-89.

5. Pulitzer DR, Reed RJ. Nerve-sheath myxoma (perineurial myxoma). Am J Dermatopathol. 1985;7:409-421.

6. Wilson ADH, Rigby H, Orlando A. Atypical cellular neurothekeoma - a diagnosis to be aware of. J Plast Reconstr Aesthetic Surg. 2008;61:186-188.

7. de Giorgi V, Alfaioli B, Franchi A, Gori A, Sestini S, Papi $\mathrm{F}$, et al. Cellular neurothekeoma in a girl: could oestrogens favour the development and growth of this rare tumour? J Eur Acad Dermatology Venereol. 2008;22:1149-1150.

8. Barnhill RL, Mihm MC. Cellular neurothekeoma. A distinctive variant of neurothekeoma mimicking nevomelanocytic tumors. Am J Surg Pathol. 1990;14:113-120.

9. Rudolph P, Schubert C. Myxoid cellular neurothekeoma. Am J Dermatopathol. 2002;24:92-93.

10. Schortinghuis J, Hille JJ, Singh S. Intraoral myxoid nerve sheath tumour. Oral Dis. 2001;7:196-199.

11. Calonje E, Wilson-Jones E, Smith NP, Fletcher CD. Cellular "neurothekeoma": an epithelioid variant of pilar leiomyoma? Morphological and immunohistochemical analysis of a series. Histopathology. 1992;20:397-404.

12. Katsourakis M, Kapranos N, Papanicolaou SI, Patrikiou A. Nerve-sheath myxoma (neurothekeoma) of the oral cavity: a case report and review of the literature. J Oral Maxillofac Surg. 1996;54:904-906.

13. Papadopoulos EJ, Cohen PR, Hebert AA. Neurothekeoma: report of a case in an infant and review of the literature. J Am Acad Dermatol. 2004;50:129-134. 